\title{
Bezafibrate prevents aging in in vitro-matured porcine oocytes
}

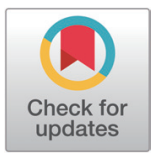

Received: Dec 22, 2020

Revised: Mar 24, 2021

Accepted: Apr 7, 2021

*Corresponding author

Xiang-Shun Cui

Department of Animal Sciences,

Chungbuk National University,

Cheongju 28644, Korea.

Tel: +82-43-261-3751

E-mail: xscui@cbnu.ac.kr

Copyright ( $\subset 2021$ Korean Society of Animal Sciences and Technology. This is an Open Access article distributed under the terms of the Creative Commons Attribution

Non-Commercial License (http:// creativecommons.org/licenses/by$\mathrm{nc} / 4.0 /$ ) which permits unrestricted non-commercial use, distribution, and reproduction in any medium, provided the original work is properly cited.

ORCID

Ju-Yeon Kim

https://orcid.org/0000-0003-3538-2830

Dongjie Zhou

https://orcid.org/0000-0002-4065-0732

Xiang-Shun Cui

https://orcid.org/0000-0003-3492-2698

Competing interests

No potential conflict of interest relevant to this article was reported.

\section{Funding sources}

This study was supported by the National Research Foundation of Korea grant funded 453 by the Korea government (Ministry of Science and Information Communication 454 Technology; 2018R1A2B6001173 and 2020R1A4A1017552) and Chungbuk National University (2019).

Acknowledgements Not applicable.

Availability of data and material Upon reasonable request, the datasets of this study can be available from the corresponding author.

\author{
Ju-Yeon Kim, Dongjie Zhou and Xiang-Shun Cui ${ }^{*}$ \\ Department of Animal Sciences, Chungbuk National University, Cheongju 28644, Korea
}

\section{Abstract}

Bezafibrate, a fibrate drug used as a lipid-lowering agent to treat hyperlipidemia, is a pan-agonist of peroxisome proliferator-activated receptor alpha. It can enhance mitochondrial fatty acid oxidation, oxidative phosphorylation, and mitochondrial biogenesis. After ovulation, oocytes may get arrested at the metaphase II (MII) stage until fertilization beyond optimal timing, which is termed as post-ovulatory aging. Post-ovulatory aging is a disease that degrades DNA, mitochondria, and oxidative system, and has a negative impact on embryo development and quality; however, the impact of bezafibrate during post-ovulatory aging has not been fully defined. In the present study, we assessed the ability of bezafibrate to prevent the progression of aging in in vitro conditions as well as the underlying mechanisms in pigs. An appropriate concentration of this drug $(50 \mu \mathrm{M})$ was added, and then oxidative stress, reactive oxygen species downstream, mitochondrial biogenesis, and mitochondrial function were analyzed via immunofluorescence staining and real-time polymerase chain reaction. Bezafibrate significantly alleviated reactive oxygen species and ameliorated glutathione production simultaneously in oocytes and embryos. Moreover, it diminished H2A.X and attenuated CASPASE 3 expression produced by oxidative stress in oocytes and embryos. Furthermore, bezafibrate remarkably improved the mitochondrial function and blastocyst quality as well as markedly reduced the mitochondria/TOM20 ratio and mtDNA copy number. The elevated PARKIN level indicated that mitophagy was induced by bezafibrate treatment after post-ovulatory aging. Collectively, these results suggest that bezafibrate beneficially affects against porcine post-ovulatory oocyte aging in porcine by its antioxidant property and mitochondrial protection. Keywords: Post-ovulatory aging, Bezafibrate, Mitochondria, Oxidative stress, PGC-1a

\section{INTRODUCTION}

Mammalian oocytes are arrested at first the meiotic prophase I during birth [1]. The prophase I oocyte undergoes maturation, including germinal vesicle breakdown and extrusion of the first polar body [2]. Following maturation, the oocyte enters secondary meiotic arrest at metaphase II (MII), wherein the optimal time for fertilization is generally within $10 \mathrm{~h}$. If this event is delayed, oocytes experience a degradative progression referred to as post-ovulatory aging (POA) [3]. POA is a common disorder characterized by reactive oxygen species (ROS) and demonstrates various reproductive phenomena of aging. So far, there has been limited discussion regarding methodologies to suppress this problem by promoting the mitochondria and oxidant system, although ROS and mitochondria are directly connected to aging. 
Authors' contributions Conceptualization: Kim JY, Cui XS. Methodology: Kim JY. Validation: Kim JY, Zhou D. Writing - original draft: Kim JY, Zhou D. Writing - review \& editing: Cui XS.

Ethics approval and consent to participate This manuscript does not require IRB/IACUC approval because there are no human and animal participants.
POA plays an important role in oocyte quality for generating successful blastocysts. Since the oocyte status determines embryogenesis [4], it is reasonable that POA causes the defects in embryonic development. Post-ovulatory aged oocytes exhibit various aberrations in their functional and biochemical mechanisms. Recent evidence suggests that POA impairs mitochondrial function [5-7], lipid peroxidation [6], and apoptosis [8] by activating caspases [7,9]. Furthermore, a survey conducted by Miao [10] dealt with the possibility of having a negative influence on porcine embryo through uncompleted mitochondrial integrity, ROS, and apoptosis [10]. Therefore, embryos originating from aged oocytes are usually of poor quality and develop slowly or are damaged in subsequent embryogenesis [7,11].

Among numerous issues, mitochondria and oxidative stress are specific mediators of the POA system. This implied that aged mitochondrial damage produces cytosolic oxidative stress [12]. Simultaneously, excessive ROS triggers apoptosis, leading to cell decomposition by releasing mitochondrial apoptogenic factors such as Caspase 3 [13]. As aforementioned, we provide an insight into blocking inferior disorder, and the point of mitochondrial regulation is special to existing studies.

Bezafibrate (Bez), one of the most widely used lipid-lowering agents to treat hyperlipidemia, is a pan-agonist of peroxisome proliferator-activated receptor ( $\operatorname{PAAR} \alpha$ ) or coactivator of peroxisome proliferator activated receptor gamma coactivator 1-alpha (PGC-1 $\alpha$ ). A key aspect of PGC- $1 \alpha$ is the control of multiple biological pathways related to energy metabolism, gluconeogenesis [14], lipid oxidation [15], mitochondrial biogenesis [15-17], mitochondrial phosphorylation [15,16], and ROS scavenging $[17,18]$ via activation of several nuclear receptor expressions. Thus, the present study aimed to assess the management effect by Bez. However, the effect of Bez on porcine oocyte and embryo after POA remains unclear.

Therefore, we designed an experiment focusing on mitochondrial and ROS disturbances affected by Bez. To clarify the mechanism of Bez on porcine oocytes and blastocysts after POA, we hypothesized that POA will be prevented by reducing the oxidative and mitochondrial disturbance cascade.

\section{MATERIALS AND METHODS}

Unless otherwise specified, all chemicals were purchased from Sigma-Aldrich (St. Louis, MO, USA), and all operations were carried out on a heating platform adjusted to $38.5^{\circ} \mathrm{C}$.

\section{Collection of porcine oocytes and in vitro maturation}

The ovaries of prepubertal gilts were used for aspiration from a local slaughterhouse (Farm Story Dodarm B\&F, Eumseong, Korea) and moved to the laboratory in $0.9 \%$ saline with $75 \mathrm{mg} / \mathrm{mL}$ penicillin $\mathrm{G}$ and $50 \mathrm{mg} / \mathrm{mL}$ streptomycin sulfate at $37^{\circ} \mathrm{C}$. Cumulus oocyte complexes (COCs) in a 3-6 $\mathrm{mm}$ diameter follicle was collected by preparing a $10 \mathrm{~mL}$ syringe connected to an 18-gauge needle, and then washed thrice with hydroxyethyl piperazine ethane sulfonic acid (HEPES) buffer. At least three layers of COCs were acquired under a stereomicroscope (Olympus, Tokyo, Japan). Thereafter, approximately 100 COCs were cultured for $48 \mathrm{hrs}$ in a 4-well dish with $500 \mu \mathrm{L}$ of TCM-199 (11150-059; Gibco, New York, NY, USA) combined with 10\% (v/v) porcine follicular fluid, $0.6 \mathrm{mM}$ L-cysteine, $0.1 \mathrm{~g} / \mathrm{L}$ sodium pyruvate, $10 \mathrm{IU} / \mathrm{mL}$ follicle-stimulating hormone (FSH), $10 \mathrm{IU} / \mathrm{mL}$ luteinizing hormone (LH), and10 $\mathrm{ng} / \mathrm{mL}$ epidermal growth factor (EGF). The in vitro maturation medium (IVM) was covered by mineral oil, the plates were balanced in a humidified atmosphere of $38.5^{\circ} \mathrm{C}$ and $5 \% \mathrm{CO}_{2}$ for $2 \mathrm{hrs}$. 


\section{In vitro aging and bezafibrate treatment}

Pipetting was repeated several times to remove the cumulus cells in HEPES added with $1 \mathrm{mg} / \mathrm{mL}$ hyaluronidase, and thereafter, oocytes which had extruded polar body were obtained. To determine whether Bez affects aging, denuded oocytes were cultured in fresh IVM medium without cysteine at $0.05,1,2.5,5,25,50,100,150$, or $200 \mu \mathrm{M} \mathrm{Bez}$, and the same amount of dimethyl sulfoxide (DMSO) was diluted as control for 24 hrs under incubation conditions.

\section{Parthenogenetic activation and in vitro culture}

Aged oocytes were washed thrice with HEPES, activated by twice direct current pulses of $110 \mathrm{~V} /$ $\mathrm{cm}$ in mannitol for $60 \mu \mathrm{s}$ and incubated in $7.5 \mu \mathrm{g} / \mathrm{mL}$ cytochalasin B for $3 \mathrm{hrs}$. Eventually, 4-cell and blastocysts were generated in in vitro compartmentalization (IVC) medium supplemented with $4 \mathrm{mg} / \mathrm{mL}$ bovine serum albumin (BSA) in PZM-5 [19] under incubating conditions at $48 \mathrm{hrs}$, or until Day 7.

\section{Glutathione and reactive oxygen species assay}

To conduct the ROS assay, oocytes and embryos were treated with $10 \mu \mathrm{M}$ 2',7'-dichlorodihydrofluorescein diacetate (D399, Invitrogen, Carlsbad, CA, USA) in poly-butylene succinate (PBS) / poly-vinyl alcohol (PVA) under incubation conditions for $30 \mathrm{~min}$, and then washed thrice PBS/ PVA.

For the glutathione (GSH) assay, oocytes and embryos were treated with $10 \mu \mathrm{M}$ 4-chloromethyl-6,8-difluoro-7-hydroxycoumarin dye (C12881, Thermo Fisher Scientific, Waltham, MA, USA) in PBS/PVA under incubation conditions for $30 \mathrm{~min}$, and then washed thrice with PBS/ PVA. Live imaging and intensity were captured using an epifluorescence microscope (Nikon, Tokyo, Japan). Furthermore, the fluorescence intensity was analyzed by Image J version $1.44 \mathrm{~g}$ software (National Institutes of Health, Bethesda, MD, USA) to quantify GSH and ROS levels.

\section{Immunofluorescence staining}

After washing three times with PBS/PVA, the oocytes and embryos were fixed in 3.7\% (w/v) paraformaldehyde (PFA) at room temperature for $30 \mathrm{~min}$. Permeabilization was carried out with $0.5 \%(\mathrm{w} / \mathrm{v})$ Triton X-100 in PBS/PVA in an incubator for $30 \mathrm{~min}$ and the cells were blocked with $1.0 \% \mathrm{BSA}$ in PBS/PVA in an incubator for $1 \mathrm{hr}$. Samples were incubated with anti-TOM20 (1:200, SC-17764, Santa Cruz Biotechnology, Dallas, TX, USA), Phospho-Histone H2A.X (1:200, \#9718, Cell Signaling Technology, Danvers, MA, USA), anti-CASPASE 3 (1:200, C8487, SigmaAldrich), or anti-PGC-1 $\alpha$ (1:200, 66369-1-Ig, Proteintech, Rosemont, IL, USA) overnight at $4^{\circ} \mathrm{C}$. After washing thrice with PBS/PVA, they were further incubated with Alexa Fluor $488 \mathrm{TM}$ Donkey anti-mouse IgG (1:100, A21202, Invitrogen), Alexa Fluor 546 TM Donkey anti-Rabbit $\operatorname{IgG}(1: 100$, A10040, Invitrogen), or Alexa Fluor 488 TM goat anti-rabbit IgG (1:100, A11034, Invitrogen) at room temperature for $1 \mathrm{hr}$. Nucleus was stained with $10 \mu \mathrm{g} / \mathrm{mL}$ Hoechst 33342 for 10 min, and washed in PBS/PVA for three times, mounted on the slides, and imaged by confocal microscope (LSM 710 META, Zeiss, Oberkochen, Germany). The image was quantified by Zen software (version 8.0, Zeiss).

\section{Active mitochondrial staining}

Oocytes and embryos were incubated in PBS/PVA with $500 \mathrm{nM}$ MitoTracker Red CMXRos (M7512, Invitrogen) for $30 \mathrm{~min}$. Samples were fixed in the PFA for $30 \mathrm{~min}$ after three times washes with IVM or IVC medium. TOM20 staining has been described in the subsection of immunofluorescence and confocal microscopy methods. 


\section{$\mathrm{JC}-1$ assay}

Blastocysts were incubated with $2.5 \mu \mathrm{M}$ 5,5',6,6'-tetrachloro-1,1',3,3'-tetraethylimidacarbocyanine iodide (JC-1) (M34152, Invitrogen) in PBS/PVA at $38.5^{\circ} \mathrm{C}$ under $5 \% \mathrm{CO}_{2}$ for $30 \mathrm{~min}$. The mitochondrial membrane potential was calculated as the ratio of red florescence to green fluorescence. Red florescence corresponds to strong activated mitochondria (JC-aggregates) and green fluorescence corresponds to less-activated mitochondria (JC-monomers). Fluorescence was observed by an epifluorescence microscope (Nikon).

\section{mtDNA copy number measurement}

Three oocytes per tube were collected with $8 \mu \mathrm{L}$ lysis buffer which contained $0.4 \mathrm{mg} / \mathrm{mL}$ proteinase K, $20 \mathrm{mM}$ Tris-HCl, $0.9 \%$ Tween 20 , and $0.9 \%$ Nonidet- 40 , and incubated at $65^{\circ} \mathrm{C}$ for $30 \mathrm{~min}$, and $95^{\circ} \mathrm{C}$ for $5 \mathrm{~min}$. Samples were diluted by sterile $\mathrm{H}_{2} \mathrm{O}$ in 1:25 ratio before analysis. Real-time quantitative polymerase chain reaction (RT-qPCR) was performed as described below.

\section{Real-time quantitative polymerase chain reaction}

DNA was obtained via RT-qPCR by using the WizPure ${ }^{\mathrm{TM}} \mathrm{qPCR}$ Master (Super Green) mix (W1731-8, Wizbiosolution, Loco Hills, NM, USA). Amplification was conducted as follows: $95^{\circ} \mathrm{C}$ for $3 \mathrm{~min}$, followed by $95^{\circ} \mathrm{C}$ for $15 \mathrm{~s}, 60^{\circ} \mathrm{C}$ for $20 \mathrm{~s}$, and $72^{\circ} \mathrm{C}$ for $15 \mathrm{~s}$ for $40 \mathrm{cycles}$, and $72^{\circ} \mathrm{C}$ for 5 $\mathrm{min}$ as a final extension. Primer sequences at target gene $N D 1$ are: forward (5'-CCT ACT GGC CGT AGC ATT CC-3') and reverse (5'-GAG GAT GTG CCT GGT CGT AG-3').

\section{Statistical analysis}

One-way analysis of variance and Student's $t$-test in the Statistical Package for the Social Sciences (SPSS) were used for data analysis. Data are expressed as mean \pm SEM. Every experiment was conducted at least thrice, and differences were considered significant when $p<0.05$ or highly significant when $p<0.001$.

\section{RESULTS}

\section{Effects of bezafibrate on development and cell number in blastocyst}

To determine the appropriate concentration, we pre-tested the effects of Bez on blastocysts after culturing parthenotes for 7 days and the blastocyst rate and quality were considered as an optimal dose response. As illustrated in Figs. $1 \mathrm{~A}$ and B, Bez had no effect on the blastocyst rate at $0.05 \mu \mathrm{M}$ (20.97 $\pm 1.41 \%), 1 \mu \mathrm{M}(23.38 \pm 3.48 \%), 2.5 \mu \mathrm{M}(21.56 \pm 1.39 \%), 5 \mu \mathrm{M}(25.03 \pm 3.32 \%), 25 \mu \mathrm{M}$ $(20.39 \pm 0.65 \%), 50 \mu \mathrm{M}(24.41 \pm 1.66 \%)$, and $100 \mu \mathrm{M}(24.44 \pm 4.68 \%)$; however, it was toxic at $150 \mu \mathrm{M}(9.39 \pm 0.25 \%)$ and $200 \mu \mathrm{M}(9.09 \pm 2.94 \%)$ compared as $0 \mu \mathrm{M}(22.31 \pm 1.31 \%, p<0.05)$. Nevertheless, the blastocyst quality, reported as the total cell number stained with Hoechst 33342, was significantly increased at $50 \mu \mathrm{M}$ treatment as compared with the control $(p<0.01$, Figs. $1 \mathrm{C}$ and D). Moreover, $50 \mu \mathrm{M}$ Bez was assumed as the base concentration for subsequent experiments. These results revealed that Bez improved the blastocyst quality in aged oocytes but not during blastocyst formation.

\section{Effects of bezafibrate on oxidative stress, DNA damage and apoptosis in oocyte}

To determine Bez-induced variations in oxidative stress in aged oocytes and embryos, the green and blue intensities were measured using 2',7'-dichlorodihydrofluorescein, and 4-chloromethyl-6,8difluoro-7-hydroxycoumarin, respectively. ROS reaction was significantly diminished in oocytes ( $p$ $<0.001$, Figs. $2 \mathrm{~A}$ and $\mathrm{B}$ ) in the presence of Bez, and GSH was naturally ameliorated in oocytes ( $p$ 
A

B
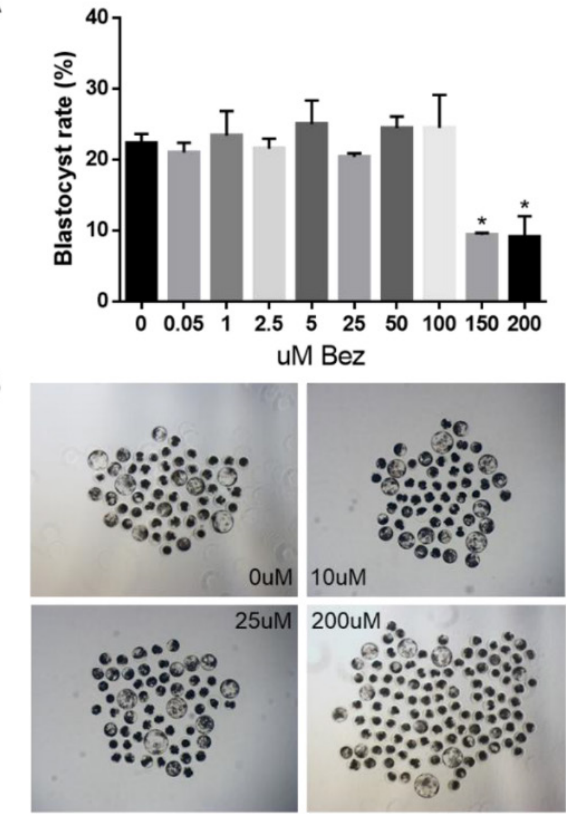

C

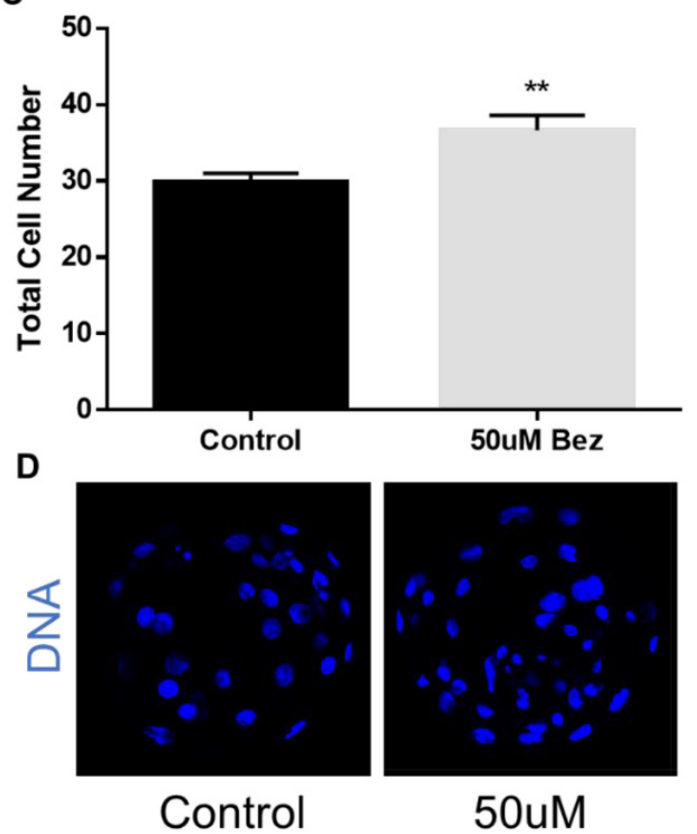

Fig. 1. Bezafibrate $(50 \mu \mathrm{M})$ improved the blastocyst quality during treatment. (A) The blastocyst formation was determined using $0 \mu \mathrm{M}(\mathrm{n}=564), 0.05 \mu \mathrm{M}$ $(n=38), 1 \mu M(n=31), 2.5 \mu M(n=28), 5 \mu M(n=28), 25 \mu M(n=49), 50 \mu M(n=220), 100 \mu M(n=55), 150 \mu M(n=11), 200 \mu M(n=47)$ concentrations. (B) Morphology captures of blastocyst formation in $0,10,25$, and $200 \mu \mathrm{M}$ concentration. (C) The total cell number of blastocyst was increased in $50 \mu \mathrm{M}(\mathrm{n}=$ 34) treatment than that in the control group $(n=88)$. (D) DNA staining with Hoechst 33342 (blue). All experiments were replicated at least thrice and data are presented as means \pm SEM. ${ }^{*} p<0.05,{ }^{* *} p<0.01$. Bez, bezafibrate.

$<0.01$, Figs. $2 \mathrm{C}$ and $\mathrm{D})$. The evidence demonstrated that Bez directly prevented oxidative stress, which was induced by POA. We next investigated whether the downstream response to oxidative stress is suppressed. H2A.X and CASPASE 3 were stained using immunofluorescence staining in aged oocytes. Subsequently, the H2A.X/Hoechst intensity ratio declined $(p<0.001$, Figs. 3A and B), and CASPASE 3 intensity was lower than that of the control in oocytes $(p<0.001$, Figs. $3 \mathrm{C}$ and D). Results revealed that, Bez decreased DNA damage, and apoptosis in oocytes against oxidative stress caused by POA.

\section{Effect of bezafibrate on peroxisome proliferator activated receptor gamma coacti-} vator 1-alpha expression, oxidative stress and apoptosis in blastocyst

To confirm whether Bez induced PGC- $1 \alpha$ successfully, we examined the protein expression of blastocysts via immunofluorescence staining after culturing aged oocytes. As hypothesized, we found that PGC-1 $\alpha$ was upregulated suitably by Bez in the blastocysts ( $p<0.01$, Figs. $4 \mathrm{~A}$ and B). Furthermore, ROS reaction was significantly diminished in blastocysts $(p<0.001$, Figs. 5A and B) in the presence of Bez, and GSH naturally also ameliorated ( $p<0.05$, Figs. 5C and D). The CASPASE 3 intensity was lower than that of the control $(p<0.05$, Figs. $5 \mathrm{E}$ and F). The evidence demonstrates that Bez-induced PGC- $1 \alpha$ prevented oxidative stress and its apoptosis during embryo development.

\section{Effects of bezafibrate on mitochondrial biogenesis in oocyte and blastocyst}

To verify that Bez improves aged mitochondrial biogenesis, active mitochondria were stained with MiotrackerRed dye, and TOM20 (mitochondrial outer membrane protein) was stained with immunofluorescence staining in aged oocytes and blastocysts. Compared with the control group, 
A

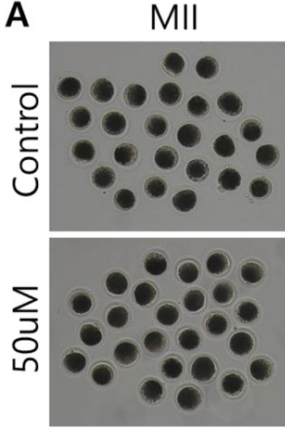

C

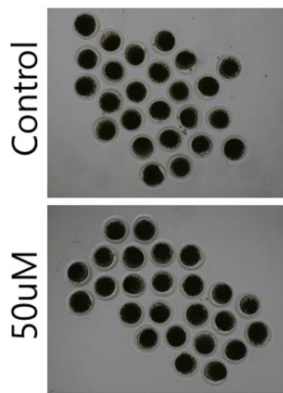

ROS

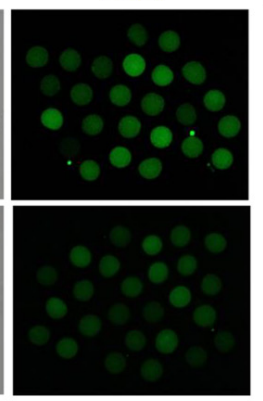

GSH

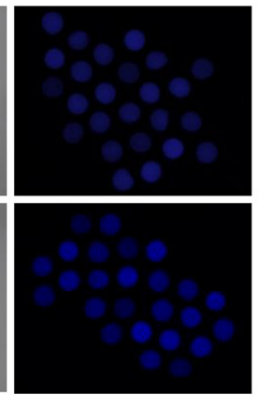

B

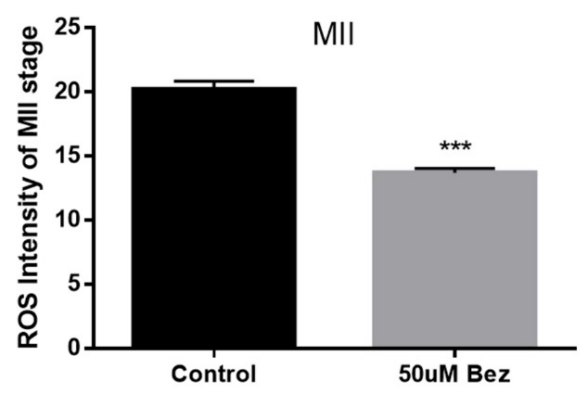

D

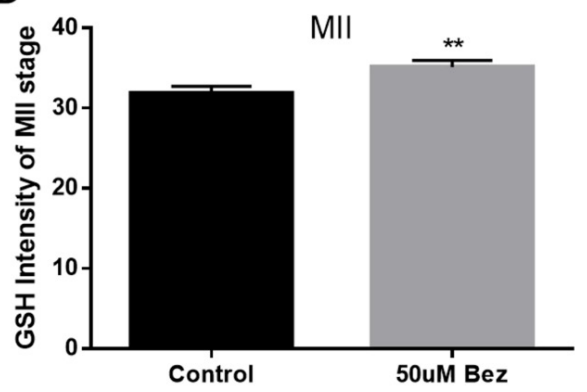

Fig. 2. Bez improved antioxidant properties in post-ovulatory aging. (A) ROS was stained with H2DCF-DA (green). (B) Relative ROS intensity of $50 \mu M$ Bez $(n=72)$ was decreased than that in the control group $(n=72)$. (C) GSH was with CellTrackerTM Blue CMF2HC (blue). (D) Relative GSH intensity of $50 \mu M$ ( $n$ $=89)$ Bez was increased than that in control group $(n=90)$. All experiments were replicated at least thrice and data are presented as means \pm SEM. ${ }^{* *} p<0.01$, ${ }^{* * *} p<0.001$. MII, metaphase II; ROS, reactive oxygen species; Bez, bezafibrate; GSH, glutathione.
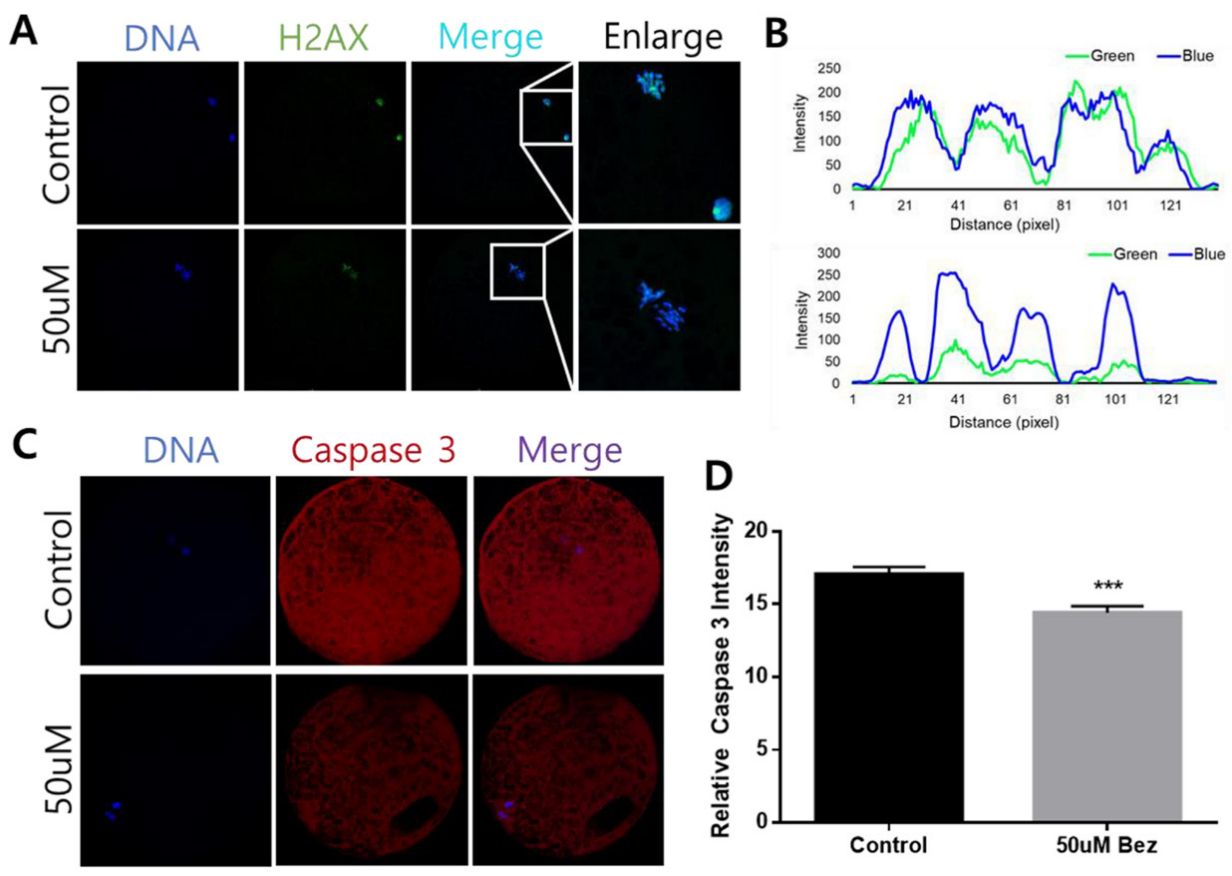

Fig. 3. Bez prevented DNA damage and apoptosis as oxidative stress during aging progress. (A) DNA was stained with Hoechst 33342 (blue), and DNA damage was stained with H2A.X antibody (green). (B) The treatment group $(n=63)$ of H2A.X/DNA ratio was decreased than that in the control group ( $n=73$ ). (C) DNA was stained with Hoechst 33342 (blue), and apoptosis was stained with CASPASE 3 antibody (red). (D) Relative intensity of CASPASE 3 was decreased in presence of Bez $(n=66)$ verses control $(n=57)$. All experiments were replicated at least thrice and data are presented as means $\pm S E M$. ${ }^{* * *} p<0.001$. Bez, bezafibrate. 
A

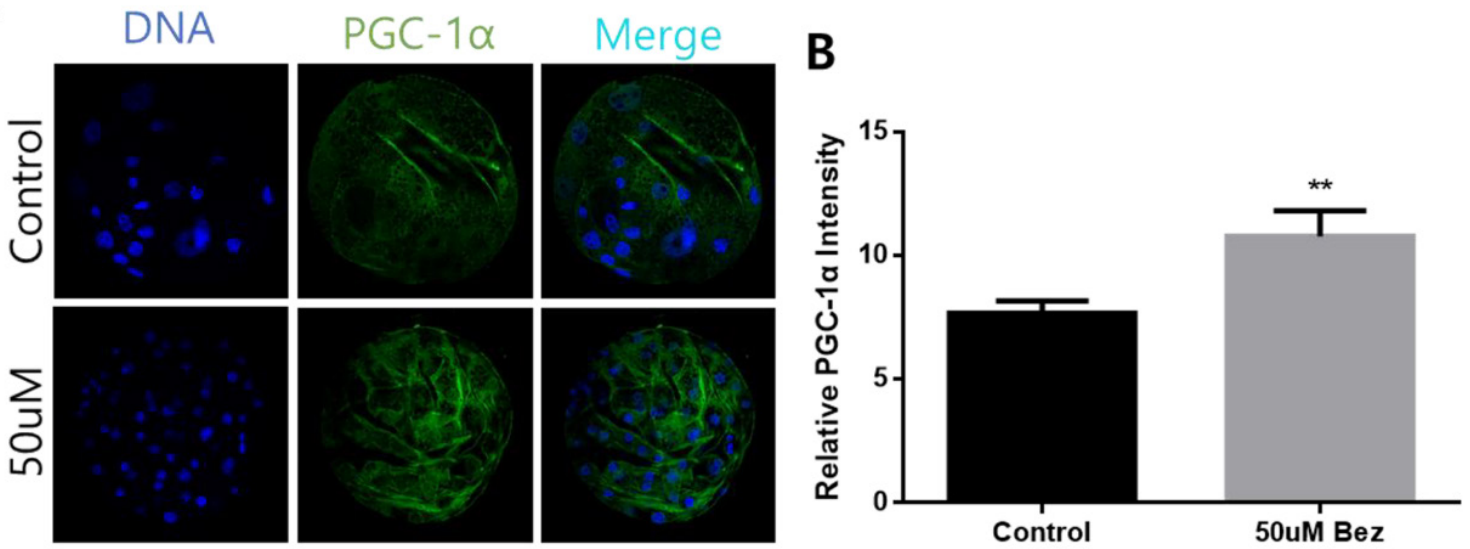

Fig. 4. Bez increased PGC-1a expression in aged blastocyst. (A) DNA was stained with Hoechst 33342 (blue), and PGC-1a was stained with PGC-1a antibody (green). (B) Relative PGC-1 $\alpha$ intensity was increased in the treatment group $(n=24)$ verses control $(n=34)$. All experiments were replicated at least thrice and data are presented as means \pm SEM. ${ }^{* *} p<0.01$. PGC-1 $\alpha$, peroxisome proliferator activated receptor gamma coactivator 1-alpha; Bez, bezafibrate.

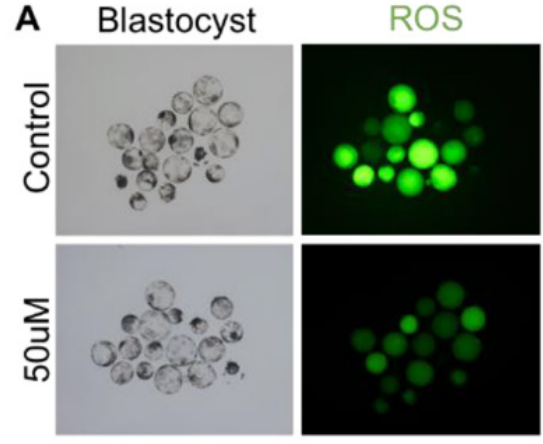

B

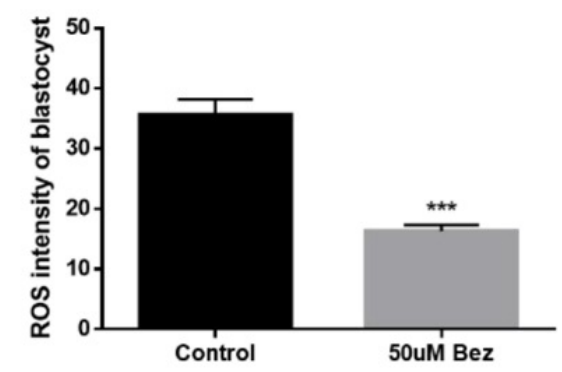

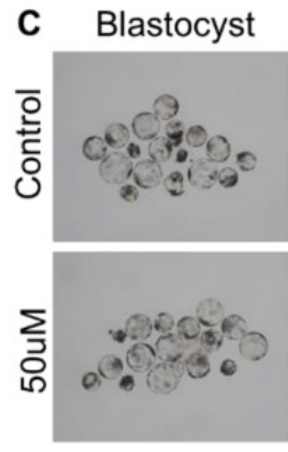

D
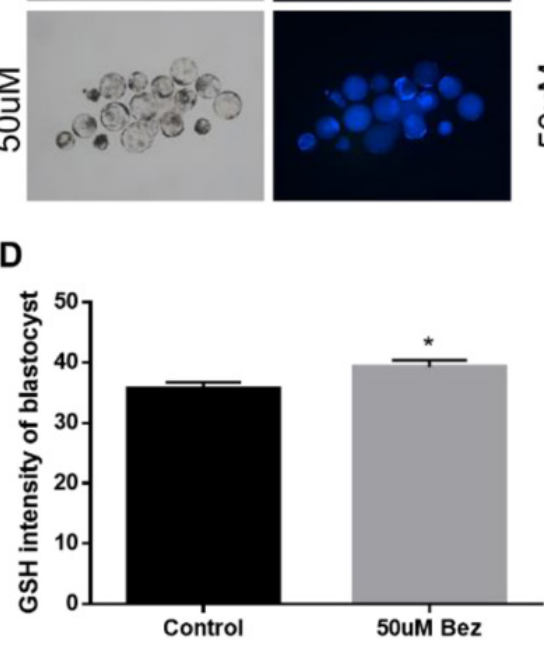

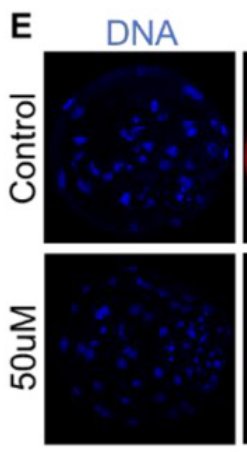

Caspase 3
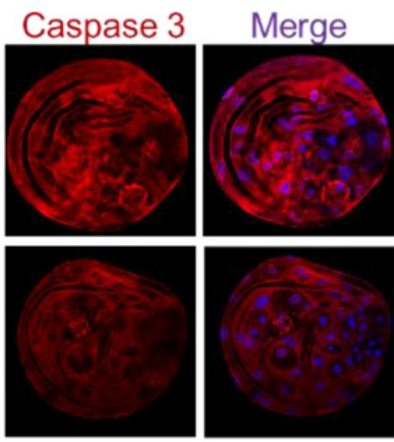

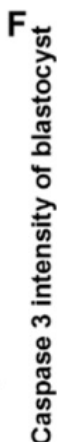

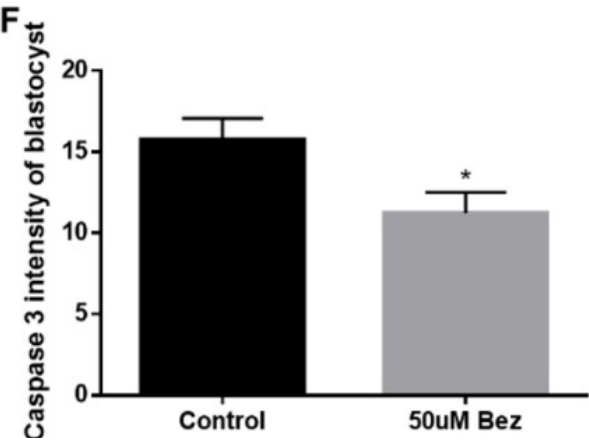

Fig. 5. Bez prevented oxidative stress and followed cascade in aged blastocysts. (A) ROS was stained with H2DCF-DA (green). (B) Relative ROS intensity of Bez $(n=46)$ was decreased than that in control group $(n=43)$. (C) GSH was with CellTrackerTM Blue CMF2HC (blue). (D) Relative GSH intensity of Bez was increased $(n=49)$ than that in control group $(n=45)$. (E) DNA was stained with Hoechst 33342 (blue), and apoptosis was stained with CASPASE 3 antibody (red). (F) Relative CASPASE 3 intensity was decreased in the presence of Bez $(n=29)$ verses control $(n=35)$. All experiments were replicated at least thrice and data are presented as means \pm SEM. ${ }^{*} p<0.05,{ }^{* \star *} p<0.001$. ROS, reactive oxygen species; GSH, glutathione; Bez, bezafibrate.

no difference was observed in protein expression such as active mitochondria, TOM20, and the MT-TOM20 ratio in oocytes. Next, while identifying the mtDNA copy number via RT-PCR, no significant difference was observed in gene expression (Figs. 6A, B, and C); however, impressive outcomes such as significantly decreased active mitochondria, increased TOM20, decreased MTTOM20 ratio, and decreased mtDNA copy number were seen in the blastocysts (Figs. 6A, D, and E).

As illustrated in Fig. 7, we examined PARKIN, a marker of mitophagy, via immunofluorescence 
staining in aged blastocysts. Relative PARKIN intensity was remarkably improved in the Bez supplemented group $(p<0.001$, Figs. 7A and B).

\section{Effects of bezafibrate on mitochondrial membrane potential in blastocyst}

Eventually, we investigated the mitochondrial membrane potential measured by the JC-1 aggregate/ monomer ratio during exposure to Bez in blastocysts. During Bez treatment, the mitochondrial membrane potential was enhanced against POA. This finding revealed that blastocysts undergo an increase in mitochondrial function $(p<0.001$, Figs. $8 \mathrm{~A}$ and $\mathrm{B})$.

A

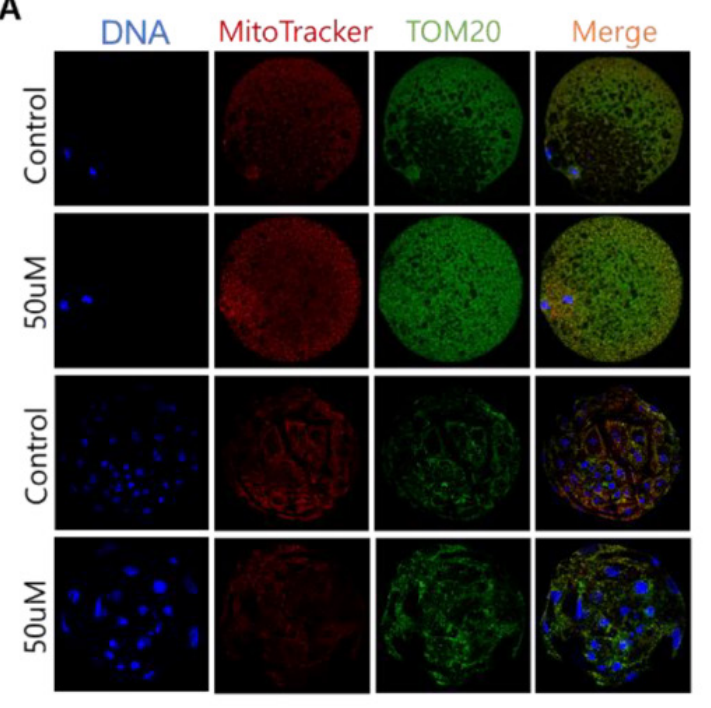

B

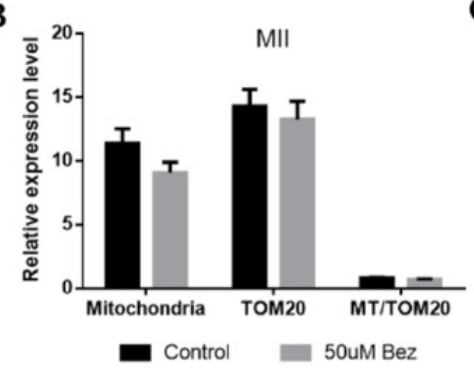

D

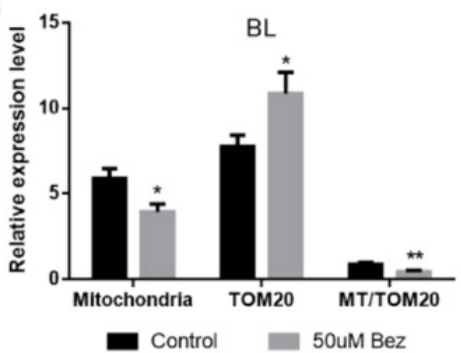

C

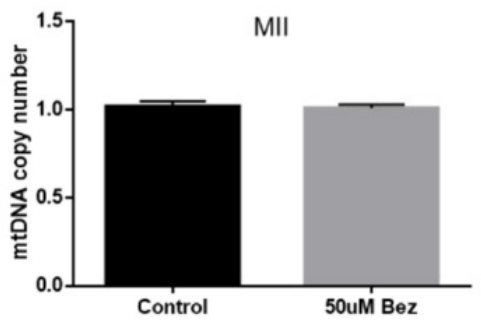

E

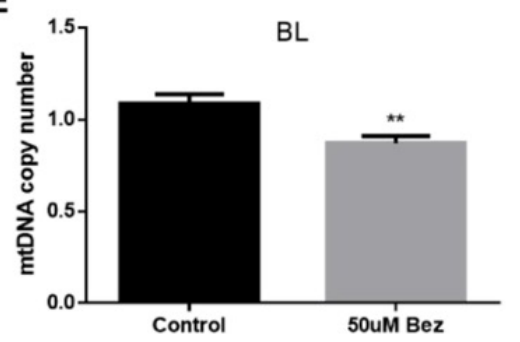

Fig. 6. Bez decreased mitochondrial biogenesis in aged blastocysts. (A) DNA was stained with Hoechst 33342 (blue), mitochondria were stained with mitotrackerRed (red), and TOM20 was stained with TOM20 antibody (green). (B) No difference was observed between control $(n=28)$ and treatment $(n=$ 28) on mitochondria, TOM20, and ratio of mitochondria/TOM20. (C) mtDNA copy number was measured via RT-PCR. No difference was observed on mtDNA copy number during treatment $(n=33)$ in oocyte $(n=48)$. (D) Mitochondria was decreased, TOM20 was increased, and ratio of mitochondria/TOM20 was decreased in the presence of Bez $(n=22)$ in aged blastocysts $(n=21)$. (E) mtDNA copy number was decreased in the presence of Bez $(n=54)$ in blastocysts $(n$ $=86$ ). All experiments were replicated at thrice times and data are presented as means \pm SEM. ${ }^{*} p<0.05,{ }^{* *} p<0.01$. MII, metaphase II; Bez, bezafibrate; RT$\mathrm{PCR}$, real-time polymerase chain reaction.

A
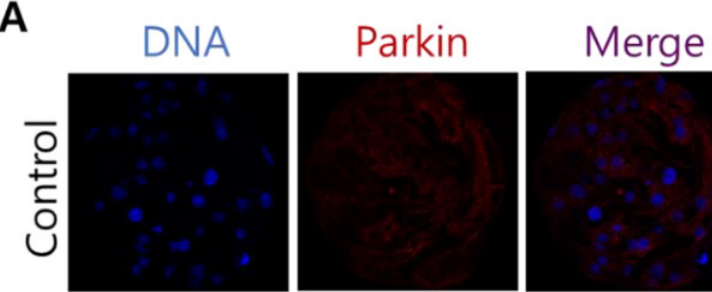

B
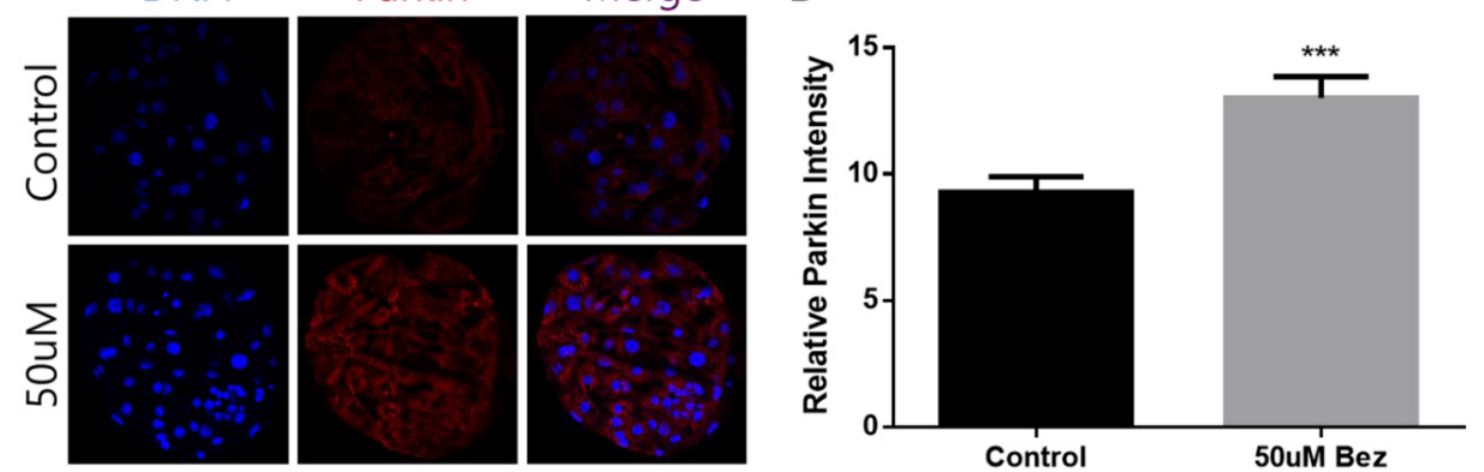

50uM Bez

Fig. 7. Bez induced mitophagy in aged blastocysts. (A) DNA was stained with Hoechst 33342 (blue), PARKIN was stained with PARKIN antibody (red). (B) Relative PARKIN intensity was induced during with treatment of Bez $(n=40)$ verses control $(n=51)$. All experiments were replicated at least thrice and data are presented as means \pm SEM. ${ }^{* * *} p<0.001$. Bez, bezafibrate. 


\section{DISCUSSION}

In recent years, $\mathrm{POA}$, has gained immense interest in research; therefore, we plan to prevent POA in in vitro porcine oocytes. POA after ovulation is an ROS-induced process that occurs when fertilization is delayed beyond the optimal period. Numerous studies reported that POA has detrimental changes, such as mitochondrial distribution, dysfunction, and ROS, leading to DNA damage and apoptosis, has and further has a negative impact on fertilization and embryo development $[5,10]$. Therefore, our research aimed for an approach to deter this adverse process in post-ovulatory aged oocytes.

Eventually, Bez, which is a coactivator of PGC- $1 \alpha$, was treated in IVM for $24 \mathrm{~h}$ to delay the side effects of aging as it is widely known to activate mitochondrial biogenesis and function [20,21]. Thus, Bez is considered as an effective retrieval treatment for aged blastocysts.

The relationship between aging and ROS has been reported in the literature $[10,12,13]$. Reproductively aged oocytes, which follow as ROS levels increase, reveal a decline in the fidelity of protective mechanisms against ROS [22,23]. A previous study has confirmed that Bez has the potential to reduce the oxidative system via ROS reduction and GSH improvement in rats [24]. Thus, we first examined the oxidative stress levels in aged porcine oocytes. We conducted at oocytes, as ROS is the main mechanism that worsen POA and mitochondria, as mentioned earlier. Our observations were consistent with this report as well as Bez, directly alleviated ROS production and induced GSH production in oocytes. Then, we conducted the blastocysts, to verify the effect of PGC- $1 \alpha$. We determined that PGC- $1 \alpha$-induced oxidative stress declined against POA with Bez. These data suggest that Bez regulates oxidative stress during aging both in a dependent and independent manner.

When subjected to oxidative stress, a high level of ROS deteriorates the oocyte quality leading to apoptosis and damaged DNA [25]. In the present study, treatment with Bez not only prevented DNA damage in oocytes but also prevented CASPASE 3 activation in oocytes and blastocysts from aging. Our results reveal that Bez can protect downstream from oxidative stress, giving the possibility of protecting POA.

Mitochondria are organelles susceptible to ROS. Abnormal mitochondrial integrity and function are regularly followed by aging-induced oxidative damage $[7,10]$. To resolve this issue, we focused on the central function of PGC1 $\alpha$, which is the detoxification of ROS [26,27]. Moreover,

A

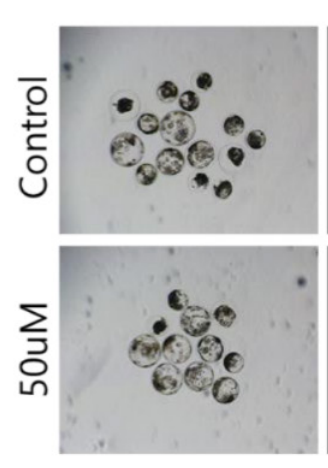

JC-1 aggregate
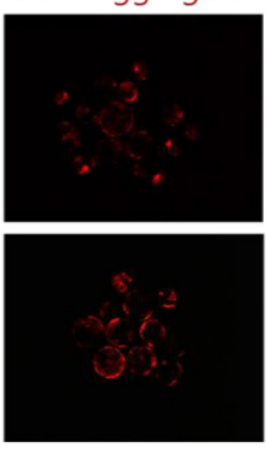
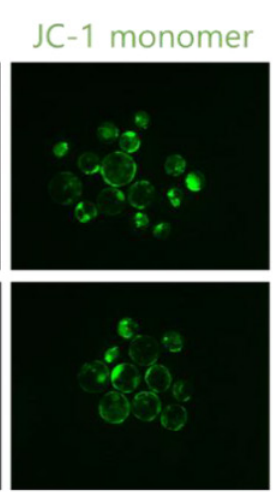

B

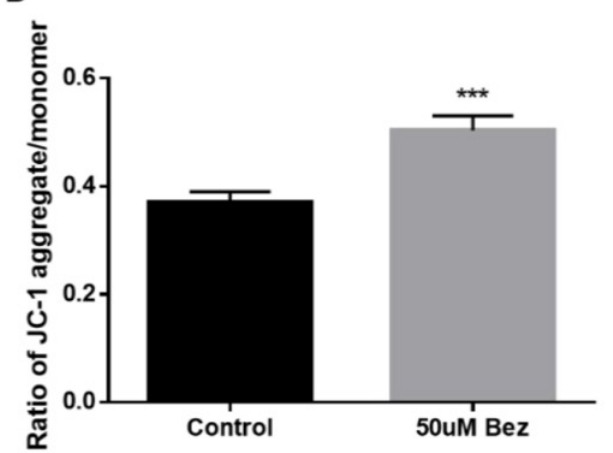

Fig. 8. Bez improved mitochondrial membrane potential in aged blastocysts. (A) Mitochondrial membrane potential was measured with JC-1 aggregate (red), and JC-1 monomer (green). (B) The ratio of JC-1 was increased during Bez treatment $(n=50)$ verses control $(n=60)$. All experiments were replicated at least thrice and data are presented as means \pm SEM. ${ }^{* * *} p<0.001$. Bez, bezafibrate. 
we illustrated mitochondrial biogenesis under Bez management in aged oocytes and blastocysts. Oocytes mtDNA rise until fertilization, the accumulation of mtDNA evenly distributed in all cells during embryogenesis [28]. This might be particularly relevant to our results, as the representation of mitochondria and mtDNA copy number were similar to controls in oocytes. Interestingly, unlike Bez, which predicts the upregulation of mitochondrial biogenesis in blastocysts, mtDNA copy number was diminished in blastocysts. Surprisingly, Bez contributed to its negative effects on the probe for mitochondria and MT/TOM20 ratio, including TOM20 expression in blastocysts. This seems to be caused by mitochondrial decomposition. Moreover, evidence reveals that nuclear respiratory factors, which are activated by Bez, promote the expression of the major members of the mitochondrial transport protein TOM20 [29].

To reveal this interesting proof, further research is required to investigate mitophagy, which triggers mitochondrial elimination. We hypothesized that mitophagy may affect mitochondrial amount. In depolarized mitochondria, PINK1 recruits PARKIN into the outer membrane of damaged mitochondrial [30]. As expected, Bez was associated with mitophagy in porcine blastocysts.

Finally, the mitochondrial membrane potential was measured for mitochondrial function in aged blastocysts. Our findings figure out that mitochondrial membrane potential was remarkably elevated despite decreased mitochondria because mitophagy led to appropriate mitochondrial maintenance. Considerably, Bez improved mitochondrial competence of aging via mitochondrial protection and antioxidative properties. Furthermore, we provided additional evidence revealing that TOM20 overexpression results in an increase in cell proliferation [31].

\section{CONCLUSION}

This project was undertaken to design a method to prevent POA and evaluate the mechanism of Bez, which has been widely used as a lipid-lowering agent, in porcine aged oocytes and blastocysts. We assume that Bez is a valuable piece of blastocyst quality that may be crucial in mitochondrial protection and reveal antioxidative properties via PGC- $1 \alpha$ during POA in pigs. Treatment with Bez during aging can diminish oxidative stress ditrectly and inditrectly through PGC-1 $\alpha$ expression. Interestingly, we observed reduced apoptosis and DNA damage. Moreover, we found that Bez increased mitochondrial function even though showed mitochondrial biogenesis was

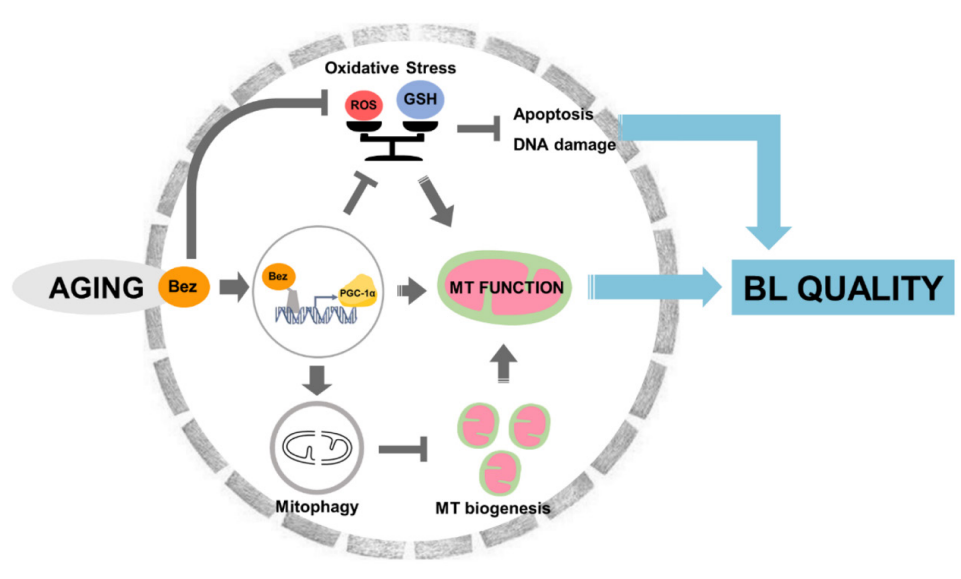

Fig. 9. Bezafibrate developmented blastocyst quality through mitochondrial protection and antioxidative properties via PGC-1a during post-ovulatory aging in pig. ROS, reactive oxygen species; GSH, glutathione; Bez, bezafibrate; PGC-1a, peroxisome proliferator activated receptor gamma coactivator 1-alpha. 
reversed and active PARKIN expression was observed. Eventually, it is obvious that blastocyst quality was improved by Bez (Fig. 9). In conclusion, a greater focus on the fact that mitochondrial function was increased despite mitophagy activity could provide interesting insights for future research.

\section{REFERENCES}

1. Tripathi A, Kumar KV, Chaube SK. Meiotic cell cycle arrest in mammalian oocytes. J Cell Physiol. 2010;223:592-600. https://doi.org/10.1002/jcp.22108

2. Sun QY, Miao YL, Schatten H. Towards a new understanding on the regulation of mammalian oocyte meiosis resumption. Cell Cycle. 2009;8:2741-7.https://doi.org/10.4161/cc.8.17.9471

3. Lord T, Aitken RJ. Oxidative stress and ageing of the post-ovulatory oocyte. Reproduction. 2013;146:R217-27.https://doi.org/10.1530/REP-13-0111

4. Mikkelsen AL, Lindenberg S. Morphology of in-vitro matured oocytes: impact on fertility potential and embryo quality. Hum Reprod. 2001;16:1714-8. https://doi.org/10.1093/humrep/16.8.1714

5. Chi MMY, Manchester JK, Yang VC, Curato AD, Strickler RC, Lowry OH. Contrast in levels of metabolic enzymes in human and mouse ova. Biol Reprod. 1988;39:295-307. https://doi. org/10.1095/biolreprod39.2.295

6. Takahashi T, Takahashi E, Igarashi H, Tezuka N, Kurachi H. Impact of oxidative stress in aged mouse oocytes on calcium oscillations at fertilization. Mol Reprod Dev. 2003;66:143-52. https://doi.org/10.1002/mrd.10341

7. Lord T, Nixon B, Jones KT, Aitken RJ. Melatonin prevents postovulatory oocyte aging in the mouse and extends the window for optimal fertilization in vitro. Biol Reprod. 2013;88:67. https://doi.org/10.1095/biolreprod.112.106450

8. Fujino Y, Ozaki K, Yamamasu S, Ito F, Matsuoka I, Hayashi E, et al. DNA fragmentation of oocytes in aged mice. Hum Reprod. 1996;11:1480-3. https://doi.org/10.1093/oxfordjournals. humrep.a019421

9. Takai Y, Matikainen T, Jurisicova A, Kim MR, Trbovich AM, Fujita E, et al. Caspase-12 compensates for lack of caspase-2 and caspase-3 in female germ cells. Apoptosis. 2007;12:791-800. https://doi.org/10.1007/s10495-006-0022-z

10. Miao Y, Zhou C, Cui Z, Zhang M, ShiYang X, Lu Y, et al. Postovulatory aging causes the deterioration of porcine oocytes via induction of oxidative stress. FASEB J. 2018;32:1328-37. https://doi.org/10.1096/f.201700908R

11. Yanagida K, Yazawa H, Katayose H, Suzuki K, Hoshi K, Sato A. Influence of oocyte preincubation time on fertilization after intracytoplasmic sperm injection. Hum Reprod. 1998;13:2223-6. https://doi.org/10.1093/humrep/13.8.2223

12. Shigenaga MK, Hagen TM, Ames BN. Oxidative damage and mitochondrial decay in aging. Proc Natl Acad Sci USA. 1994;91:10771-8. https://doi.org/10.1073/pnas.91.23.10771

13. Redza-Dutordoir M, Averill-Bates DA. Activation of apoptosis signalling pathways by reactive oxygen species. Biochim Biophys Acta Mol Cell Res. 2016;1863:2977-92. https://doi. org/10.1016/j.bbamcr.2016.09.012

14. Yoon JC, Puigserver P, Chen G, Donovan J, Wu Z, Rhee J, et al. Control of hepatic gluconeogenesis through the transcriptional coactivator PGC-1. Nature. 2001;413:131-8. https://doi. org/10.1038/35093050

15. Nikolić N, Rhedin M, Rustan AC, Storlien L, Thoresen GH, Strömstedt M. Overexpression of PGC-1 $\alpha$ increases fatty acid oxidative capacity of human skeletal muscle cells. Biochem Res Int. 2012;2012:714074. https://doi.org/10.1155/2012/714074 
16. LeBleu VS, O'Connell JT, Gonzalez Herrera KN, Wikman H, Pantel K, Haigis MC, et al. PGC-1 $\alpha$ mediates mitochondrial biogenesis and oxidative phosphorylation in cancer cells to promote metastasis. Nat Cell Biol. 2014;16:992-1003. https://doi.org/10.1038/ncb3039

17. Johri A, Calingasan NY, Hennessey TM, Sharma A, Yang L, Wille E, et al. Pharmacologic activation of mitochondrial biogenesis exerts widespread beneficial effects in a transgenic mouse model of Huntington's disease. Hum Mol Genet. 2012;21:1124-37. https://doi.org/10.1093/ $\mathrm{hmg} / \mathrm{ddr} 541$

18. Baldelli S, Aquilano K, Ciriolo MR. PGC- $1 \alpha$ buffers ROS-mediated removal of mitochondria during myogenesis. Cell Death Dis. 2014;5:e1515. https://doi.org/10.1038/cddis.2014.458

19. Yoshioka K, Suzuki C, Onishi A. Defined system for in vitro production of porcine embryos using a single basic medium.J Reprod Dev. 2008;54:208-13. https://doi.org/10.1262/jrd.20001

20. Bonnefont JP, Bastin J, Behin A, Djouadi F. Bezafibrate for an inborn mitochondrial beta-oxidation defect. N Eng1 J Med. 2009;360:838-40. https://doi.org/10.1056/NEJMc0806334

21. Steele H, Gomez-Duran A, Pyle A, Hopton S, Newman J, Stefanetti RJ, et al. Metabolic effects of bezafibrate in mitochondrial disease. EMBO Mol Med. 2020;12:e11589. https://doi. org/10.15252/emmm.201911589

22. Sasaki H, Hamatani T, Kamijo S, Iwai M, Kobanawa M, Ogawa S, et al. Impact of oxidative stress on age-associated decline in oocyte developmental competence. Front Endocrinol. 2019;10:811. https://doi.org/10.3389/fendo.2019.00811

23. Igarashi $H$, Takahashi $T$, Nagase $S$. Oocyte aging underlies female reproductive aging: biological mechanisms and therapeutic strategies. Reprod Med Biol. 2015;14:159-69. https://doi. org/10.1007/s12522-015-0209-5

24. Grings M, Moura AP, Parmeggiani B, Pletsch JT, Cardoso GMF, August PM, et al. Bezafibrate prevents mitochondrial dysfunction, antioxidant system disturbance, glial reactivity and neuronal damage induced by sulfite administration in striatum of rats: implications for a possible therapeutic strategy for sulfite oxidase deficiency. Biochim Biophys Acta Mol Basis Dis. 2017;1863:2135-48. https://doi.org/10.1016/j.bbadis.2017.05.019

25. Agarwal A, Gupta S, Sharma RK. Role of oxidative stress in female reproduction. Reprod Biol Endocrinol. 2005;3:28. https://doi.org/10.1186/1477-7827-3-28

26. St-Pierre J, Drori S, Uldry M, Silvaggi JM, Rhee J, Jäger S, et al. Suppression of reactive oxygen species and neurodegeneration by the PGC-1 transcriptional coactivators. Cell. 2006;127:397408. https://doi.org/10.1016/j.cell.2006.09.024

27. St-Pierre J, Lin J, Krauss S, Tarr PT, Yang R, Newgard CB, et al. Bioenergetic analysis of peroxisome proliferator-activated receptor $\gamma$ coactivators $1 \alpha$ and $1 \beta$ (PGC- $1 \alpha$ and PGC-1 $\beta$ ) in muscle cells.J Biol Chem. 2003;278:26597-603. https://doi.org/10.1074/jbc.M301850200

28. Cecchino GN, Seli E, Alves da Motta EL, García-Velasco JA. The role of mitochondrial activity in female fertility and assisted reproductive technologies: overview and current insights. Reprod Biomed Online. 2018;36:686-97.https://doi.org/10.1016/j.rbmo.2018.02.007

29. Blesa JR, Prieto-Ruiz JA, Hernández JM, Hernández-Yago J. NRF-2 transcription factor is required for human TOMM20 gene expression. Gene. 2007;391:198-208. https://doi. org/10.1016/j.gene.2006.12.024

30. Narendra DP, Youle RJ. Targeting mitochondrial dysfunction: role for PINK1 and Parkin in mitochondrial quality control. Antioxid Redox Signal. 2011;14:1929-38. https://doi. org/10.1089/ars.2010.3799

31. Park SH, Lee AR, Choi K, Joung S, Yoon JB, Kim S. TOMM20 as a potential therapeutic target of colorectal cancer. BMB Rep. 2019;52:712-7. https://doi.org/10.5483/BMBRep.2019.52.12.249 